TRANSACTIONS OF THE

AMERICAN MATHEMATICAL SOCIETY

Volume 355, Number 8, Pages 3079-3098

S 0002-9947(03)03313-0

Article electronically published on April 25, 2003

\title{
EXPLICIT LOWER BOUNDS FOR RESIDUES AT $s=1$ OF DEDEKIND ZETA FUNCTIONS AND RELATIVE CLASS NUMBERS OF CM-FIELDS
}

\author{
STÉPHANE LOUBOUTIN \\ Dedicated to Jacqueline G.
}

\begin{abstract}
Let $S$ be a given set of positive rational primes. Assume that the value of the Dedekind zeta function $\zeta_{K}$ of a number field $K$ is less than or equal to zero at some real point $\beta$ in the range $\frac{1}{2}<\beta<1$. We give explicit lower bounds on the residue at $s=1$ of this Dedekind zeta function which depend on $\beta$, the absolute value $d_{K}$ of the discriminant of $K$ and the behavior in $K$ of the rational primes $p \in S$. Now, let $k$ be a real abelian number field and let $\beta$ be any real zero of the zeta function of $k$. We give an upper bound on the residue at $s=1$ of $\zeta_{k}$ which depends on $\beta, d_{k}$ and the behavior in $k$ of the rational primes $p \in S$. By combining these two results, we obtain lower bounds for the relative class numbers of some normal CM-fields $K$ which depend on the behavior in $K$ of the rational primes $p \in S$. We will then show that these new lower bounds for relative class numbers are of paramount importance for solving, for example, the exponent-two class group problem for the non-normal quartic CM-fields. Finally, we will prove Brauer-Siegel-like results about the asymptotic behavior of relative class numbers of CM-fields.
\end{abstract}

The main results arrived at in this paper are Theorems 1, 14, 22 and 26.

\section{LOWER BOUNDS FOR RESIDUES OF ZETA FUNCTIONS}

Let $c>0$ be given (to be selected below). It has long been known that Hecke's integral representations of Dedekind zeta functions $\zeta_{K}$ of number fields $K$ can be used to obtain lower bounds for their residues $\kappa_{K}$ at $s=1$ of the type

$$
1-\left(c / \log d_{K}\right) \leq \beta<1 \text { and } \zeta_{K}(\beta) \leq 0 \text { imply } \kappa_{K} \geq(1-\beta) d_{K}^{(\beta-1) / 2}(1+o(1)),
$$

where $o(1)$ is an error term that approaches zero as $d_{K} \rightarrow \infty$ provided that $K$ ranges over number fields of a given degree (e.g. see [Lou2, Proposition A]. See also [Lan, Chapter XVI, Section 2, Lemma 3, p. 323] for a weaker result). Notice that the best lower bound one can deduce (for $\beta=1-\left(2 / \log d_{K}\right)$ ) is of the type

$$
\zeta_{K}\left(1-\left(2 / \log d_{K}\right)\right) \leq 0 \text { implies } \kappa_{K} \geq \frac{2}{e \log d_{K}}(1+o(1)) .
$$

The first aim of this paper is to prove Theorem 1 below, which not only provides a nice treatment of this error term (by simply getting rid of it!) but also allows us to obtain lower bounds for these residues which depend on the behavior in $K$ of a

Received by the editors April 23, 2002 and, in revised form, January 6, 2003.

2000 Mathematics Subject Classification. Primary 11R42; Secondary 11R29.

Key words and phrases. Dedekind zeta functions, CM-field, relative class number. 
finite set $S$ of rational primes. Let us first set some notation. If $K$ is an algebraic number field and $S$ is any finite set of positive rational primes, we define

$$
\Pi_{K}(S):=\prod_{p \in S} \prod_{\mathcal{P} \mid p}\left(1-(N(\mathcal{P}))^{-1}\right)^{-1} \geq 1
$$

(product of Euler's factors of the Dedekind zeta function of $K$ ) and

$$
\Lambda_{S}:=\prod_{p \in S}\left(1+p^{-1 / 2}\right)^{4} \geq 1
$$

with the convention $\Pi_{K}(\emptyset)=\Lambda_{\emptyset}=1$. Our first result is as follows:

\section{Theorem 1.}

(1) Let $m \geq 1$ be a positive integer. There exists $\rho_{2 m}$ effective such that for any finite set $S$ of primes and any totally imaginary number field $K$ of degree $2 n \geq 2 m$ and root discriminant $\rho_{K}:=d_{K}^{1 / 2 n} \geq \rho_{2 m} \Lambda_{S}$ we have

$$
\kappa_{K} \geq \frac{1}{2}(1-\beta) d_{K}^{(\beta-1) / 2} \Pi_{K}(S)
$$

if $\zeta_{K}(\beta) \leq 0$ for some $\beta \in\left[1-\left(2 / \log d_{K}\right), 1\right)$.

(2) Let $m \geq 1$ be a positive integer. Let $S$ be any given finite set of primes. There exists $\rho_{2 m, S}$ effective such that for any totally imaginary number field $K$ of degree $2 n \geq 2 m$ and root discriminant $\rho_{K}:=d_{K}^{1 / 2 n} \geq \rho_{2 m, S}$ we have

$$
\kappa_{K} \geq(1-\beta) d_{K}^{(\beta-1) / 2} \Pi_{K}(S)
$$

if $\zeta_{K}(\beta) \leq 0$ for some $\beta \in\left[1-\left(2 / \log d_{K}\right), 1-\left(1 / \log d_{K}\right)\right]$.

(3) We may take $\rho_{12}=\rho_{12, \emptyset}=2 \pi^{2}$ and for smaller values of $m$ we may take $\rho_{2 m}$ and $\rho_{2 m, S}$ for $S=\emptyset$ and $S=\{2\}$ as given in Table 1:

\begin{tabular}{|c|ccccccc|}
\multicolumn{10}{c|}{ Table 1} \\
\hline $2 n \geq 2 m=$ & 2 & 4 & 6 & 8 & 10 & 12 & $\infty$ \\
$\rho_{2 m}=$ & 270 & 41 & 26 & 22 & 21 & $2 \pi^{2}$ & $2 \pi^{2}$ \\
$\rho_{2 m, \emptyset}=$ & 2600 & 50 & 25 & 20 & $2 \pi^{2}$ & $2 \pi^{2}$ & $2 \pi^{2}$ \\
$\rho_{2 m,\{2\}}=$ & 36000 & 650 & 295 & 222 & 194 & 181 & $2 \pi^{2} \Lambda_{\{2\}}=167.63 \ldots$ \\
\hline
\end{tabular}

(4) Let $K$ be a totally imaginary number field of degree $2 n>2$ and root discriminant $\rho_{K} \geq 32 \pi^{2} \Lambda_{\{2\}}=2682.208 \cdots$. Assume that $\zeta_{K}(\beta) \leq 0$ for some $\beta \in\left[1-\left(2 / \log d_{K}\right), 1\right)$. Then,

$$
\kappa_{K} \geq(1-\beta) d_{K}^{(\beta-1) / 2} .
$$

Proof. See Section 2 below.

We could have stated this result in the more general setting of the not necessarily totally imaginary number fields. However, we only aim at using it for obtaining good lower bounds for relative class numbers of CM-fields. Notice that, contrary to our previous lower bounds given in [Lou2, Proposition A], our present lower bounds (11) and (21) do not depend on any pesky error factor

$$
\epsilon_{K}=\max \left(1-\left(2 \pi n / \rho_{K}^{\beta}\right), \frac{2}{5} \exp \left(-2 \pi n / \rho_{K}\right)\right),
$$

which for a given $n$ approaches 1 as $d_{K} \rightarrow \infty$, but which approaches 0 as $n \rightarrow \infty$ as $K$ ranges over CM-fields of bounded root discriminants. Moreover, the real draw of these lower bounds (11) and (2) is that the Euler factors $\Pi_{K}(S)$ being always greater 
than or equal to one, these bounds can be considerably better than the ones without the factor $\Pi_{K}(S)$ given in [Lou2, Proposition A]. For example, if $S=\{2\}$ and 2 splits completely in $K$, then $\Pi_{K}(S)=4^{n}$. We also refer the reader to [Hof, Lemma 4] and [Sta3 Lemma 4] where other similar but less satisfactory lower bounds for $\kappa_{K}$ are proved (in the case that $S=\emptyset$ ).

\section{Proof of Theorem 1}

Let $K$ be a totally imaginary number field of degree $2 n \geq 2$. Let $\zeta_{K}(s)$ and $d_{K}$ be the the Dedekind zeta function and the absolute value of the discriminant of $K$, and set $A_{K}=\sqrt{d_{K} /(2 \pi)^{2 n}}=\left(\rho_{K} / 2 \pi\right)^{n}, F_{K}(s)=A_{K}^{s} \Gamma^{n}(s) \zeta_{K}(s)$ and $\lambda_{K}=$ $\operatorname{Res}_{s=1}\left(F_{K}\right)=A_{K} \kappa_{K}$. Let

$$
H_{n}(x)=\frac{1}{2 \pi i} \int_{\Re(z)=\alpha} \Gamma^{n}(z) x^{-z} d z \quad(\alpha>1 \text { and } x>0)
$$

be the inverse Mellin transform of $\Gamma^{n}(s)$. Hence, $H_{n}(x)>0$ for $x>0$. Let $S_{K}(x)$ be the inverse Mellin tansform of $F_{K}(s)$. For $x>0$ we have

$$
S_{K}(x)=\frac{1}{2 \pi i} \int_{\Re(z)=\alpha} F_{K}(z) x^{-z} d z=\sum_{\mathcal{I}} H_{n}\left(x N(\mathcal{I}) / A_{K}\right)
$$

(where $\mathcal{I}$ ranges over the nonzero integral ideals of $K$ ). Now, by shifting the vertical line of integration $\Re(z)=\alpha>1$ to the left to the vertical line $\Re(z)=1-\alpha<0$, by using the functional equation $F_{K}(1-z)=F_{K}(z)$ to come back to the vertical line of integration $\Re(z)=\alpha$ and by noticing that we pick up only two poles, a simple pole of residue $\lambda_{K}$ at $z=1$ and a simple pole of residue $-\lambda_{K}$ at $z=0$, we obtain that $S_{K}(x)$ satisfies the following functional equation:

$$
S_{K}(1 / x)=\lambda_{K} x-\lambda_{K}+x S_{K}(x) .
$$

Using this functional equation and the fact that $F_{K}(s)$ is the Mellin transform of $S_{K}(x)$, we obtain:

$$
\begin{aligned}
F_{K}(s)=\int_{0}^{\infty} S_{K}(x) x^{s} \frac{d x}{x} & =\int_{1}^{\infty} S_{K}(1 / x) x^{-s} \frac{d x}{x}+\int_{1}^{\infty} S_{K}(x) x^{s} \frac{d x}{x} \\
& =\frac{\lambda_{K}}{s(s-1)}+\int_{1}^{\infty} S_{K}(x)\left(x^{s}+x^{1-s}\right) \frac{d x}{x}
\end{aligned}
$$

and

$$
F_{K}(s)=\frac{\lambda_{K}}{s(s-1)}+\sum_{\mathcal{I}} \int_{1}^{\infty} H_{n}\left(x N(\mathcal{I}) / A_{K}\right)\left(x^{s}+x^{1-s}\right) \frac{d x}{x}
$$

(where $\mathcal{I}$ ranges over the nonzero integral ideals of $K$ ), which is nothing but the Hecke integral representation of $\zeta_{K}(s)$, in another guise (see [Lan, Chapter XIII, Section 3, Theorem 3, p. 260]). Let $S$ be a finite set of distinct rational primes. Set $\mathcal{S}=\{\mathcal{I} ; p \mid N(\mathcal{I}) \Rightarrow p \in S\}$ and

$$
\zeta_{S}(s)=\sum_{\mathcal{I} \in \mathcal{S}}(N(\mathcal{I}))^{-s}=\prod_{p \in S} \prod_{\mathcal{P} \mid p}\left(1-(N(\mathcal{P}))^{-s}\right)^{-1}
$$


(hence, $\zeta_{S}(1)=\Pi_{K}(S)$ ). Since $H_{n}(x)>0$ for $x>0$, for $1-\alpha<s<\alpha$ we have

$$
\begin{aligned}
& F_{K}(s) \\
& \quad \geq \frac{\lambda_{K}}{s(s-1)}+\sum_{\mathcal{I} \in \mathcal{S}} \int_{1}^{\infty} H_{n}\left(x N(\mathcal{I}) / A_{K}\right)\left(x^{s}+x^{1-s}\right) \frac{d x}{x} \\
& =\frac{\lambda_{K}}{s(s-1)}+\sum_{\mathcal{I} \in \mathcal{S}} \frac{1}{2 \pi i} \int_{\Re(z)=\alpha} \Gamma^{n}(z)\left(A_{K} / N(\mathcal{I})\right)^{z}\left(\frac{1}{z-s}+\frac{1}{z-(1-s)}\right) d z \\
& =\frac{\lambda_{K}}{s(s-1)}+\frac{1}{2 \pi i} \int_{\Re(z)=\alpha} \Gamma^{n}(z) A_{K}^{z} \zeta_{S}(z)\left(\frac{1}{z-s}+\frac{1}{z-(1-s)}\right) d z .
\end{aligned}
$$

Since $0<s<1$ and $\zeta_{K}(s) \leq 0$ imply $F_{K}(s) \leq 0$, we obtain:

Lemma 2. Fix $\alpha>1$. Assume that $\zeta_{K}(\beta) \leq 0$ for some $\beta \in(0,1)$. Then,

$$
\kappa_{K} \geq \frac{\beta(1-\beta)}{2 \pi i} \int_{\Re(z)=\alpha} \Gamma^{n}(z) A_{K}^{z-1} \zeta_{S}(z)\left(\frac{1}{z-\beta}+\frac{1}{z-(1-\beta)}\right) d z .
$$

From now on, we assume that $\frac{1}{2}<\beta<1$. We set

$$
\begin{gathered}
f_{n}(\beta)=\beta(2 \pi)^{n(1-\beta)} \Gamma^{n}(\beta), \\
M(\beta)=\sup _{\Re(s)=1 / 2}\left|\frac{1}{s-\beta}+\frac{1}{s-(1-\beta)}\right|=\sup _{-\infty<t<\infty} \frac{2|t|}{\left(\beta-\frac{1}{2}\right)^{2}+t^{2}}=\frac{2}{2 \beta-1}
\end{gathered}
$$

and

$$
I_{n}=\frac{1}{2 \pi} \int_{-\infty}^{\infty}\left|\Gamma\left(\frac{1}{2}+i t\right)\right|^{n} d t=\frac{1}{\pi} \int_{0}^{\infty}\left(\frac{\pi}{\cosh (\pi t)}\right)^{n / 2} d t=\pi^{\frac{n}{2}-2} J_{n},
$$

where

$$
J_{n}=\int_{0}^{\pi / 2} \sin ^{n / 2-1}(T) d T
$$

decreases towards zero as $n \geq 1$ goes to infinity (use $\Gamma(s) \Gamma(1-s)=\pi / \sin (\pi s$ ) to obtain $|\Gamma(1 / 2+i t)|^{2}=\pi / \cosh (\pi t)$, and set $\left.\cosh (\pi t)=1 / \sin T\right)$. Notice that $J_{n+4}=\frac{n}{n+2} J_{n}$ for $n \geq 1$ and that $J_{2}=\pi / 2$ and $J_{4}=1$.

In Lemma 2, we shift the vertical line of integration $\Re(z)=\alpha>1$ leftwards to the vertical line $\Re(z)=1 / 2$. We pick up only one residue, at $z=\beta$, and obtain:

$$
\begin{aligned}
\kappa_{K} & \geq \beta(1-\beta)\left(\Gamma^{n}(\beta) A_{K}^{\beta-1} \zeta_{S}(\beta)-M(\beta) \zeta_{S}(1 / 2) A_{K}^{-1 / 2} I_{n}\right) \\
& \geq(1-\beta) d_{K}^{(\beta-1) / 2} \zeta_{S}(1)\left(f_{n}(\beta)-\frac{2 \beta d_{K}^{(1-\beta) / 2}}{2 \beta-1} \frac{\zeta_{S}(1 / 2)}{\zeta_{S}(1)}\left(\frac{2 \pi^{2}}{\rho_{K}}\right)^{n / 2} I_{n}\right) \\
& \geq(1-\beta) d_{K}^{(\beta-1) / 2} \Pi_{K}(S)\left(f_{n}(\beta)-\frac{2 \beta d_{K}^{(1-\beta) / 2} J_{n}}{(2 \beta-1) \pi^{2}}\left(\frac{2 \pi^{2} \Lambda_{S}}{\rho_{K}}\right)^{n / 2}\right),
\end{aligned}
$$

for

$$
\frac{\zeta_{S}(1 / 2)}{\zeta_{S}(1)}=\prod_{\mathcal{P} \mid p}\left(1+(N(\mathcal{P}))^{-1 / 2}\right) \leq\left(1+p^{-1 / 2}\right)^{2 n}=\Lambda_{S}^{n / 2}
$$

and $\zeta_{S}(1)=\Pi_{K}(S)$. 
Lemma 3. Let $\gamma=0.577215 \cdots$ denote Euler's constant and set $f_{n}(\beta):=$ $\beta(2 \pi)^{n(1-\beta)} \Gamma^{n}(\beta)$. In the range $0<\beta \leq 1$, it follows that

$$
f_{n}(\beta) \geq 1-(1-\beta) f_{n}^{\prime}(1)=1+n(1-\beta)\left(\gamma+\log (2 \pi)-\frac{1}{n}\right) \geq 1 .
$$

Proof. Since $f_{n}(\beta)$ is positive and log-convex in the range $\beta>0$ (use the infinite product of the $\Gamma$-function), $f_{n}$ is convex in the same range.

Using Lemma 3, noticing that $1 / 2<1-\left(2 / \log d_{K}\right) \leq \beta<1$ implies $\beta /(2 \beta-1) \leq$ $\left(n \log \rho_{K}-1\right) /\left(n \log \rho_{K}-2\right)$ and $d_{K}^{(1-\beta) / 2} \leq e$, and noticing that $1-\left(2 / \log d_{K}\right) \leq$ $\beta \leq 1-\left(1 / \log d_{K}\right)$ implies $d_{K}^{(1-\beta) / 2} /(1-\beta) \leq \sqrt{e} \log d_{K}$, we finally obtain:

Proposition 4. Let $K$ be a totally imaginary number field of degree $2 n \geq 2$, and assume that $d_{K} \geq e^{4}$.

(1) Assume that $\zeta_{K}(\beta) \leq 0$ for some $\beta \in\left[1-\left(2 / \log d_{K}\right), 1\right)$. Then,

$$
\kappa_{K} \geq(1-\beta) d_{K}^{(\beta-1) / 2} \Pi_{K}(S)\left(1-\frac{n \log \rho_{K}-1}{n \log \rho_{K}-2} \frac{2 e J_{n}}{\pi^{2}}\left(\frac{2 \pi^{2} \Lambda_{S}}{\rho_{K}}\right)^{n / 2}\right) .
$$

(2) Assume that $\zeta_{K}(\beta) \leq 0$ for some $\beta \in\left[1-\left(2 / \log d_{K}\right), 1-\left(1 / \log d_{K}\right)\right]$. Then,

$$
\kappa_{K} \geq(1-\beta) d_{K}^{(\beta-1) / 2} \Pi_{K}(S)\left(1+n(1-\beta) S_{K}\right)
$$

where

$$
S_{K}=\gamma+\log (2 \pi)-\frac{1}{n}-\frac{n \log \rho_{K}-1}{n \log \rho_{K}-2} \frac{4 \sqrt{e} J_{n}}{\pi^{2}} R_{K}
$$

and where

$$
R_{K}=\left(\frac{2 \pi^{2} \Lambda_{S}}{\rho_{K}}\right)^{n / 2} \log \rho_{K}
$$

decreases with $\rho_{K}$ in the range $\rho_{K} \geq e^{2 / n}$, i.e., in the range $d_{K} \geq e^{4}$.

Now, we are in a position to complete the proof of Theorem 1 .

(1) Assume that $n \geq 6$ and $\rho_{K} \geq 2 \pi^{2} \Lambda_{S} \geq 2 \pi^{2}$. We have $J_{n} \leq J_{6}=\pi / 4$, $\left(n \log \rho_{K}-1\right) /\left(n \log \rho_{K}-2\right) \leq\left(6 \log \left(2 \pi^{2}\right)-1\right) /\left(6 \log \left(2 \pi^{2}\right)-2\right)$, and we obtain

$$
\frac{n \log \rho_{K}-1}{n \log \rho_{K}-2} \frac{2 e J_{n}}{\pi^{2}}\left(\frac{2 \pi^{2} \Lambda_{S}}{\rho_{K}}\right)^{n / 2} \leq \frac{6 \log \left(2 \pi^{2}\right)-1}{6 \log \left(2 \pi^{2}\right)-2} \frac{e}{2 \pi}<\frac{1}{2}
$$

and (4) yields (11) with $\rho_{12}=2 \pi^{2}$. Moreover, if $S=\emptyset$, then $\Lambda_{S}=1$ and

$$
S_{K} \geq \gamma+\log (2 \pi)-\frac{1}{6}-\frac{6 \log \left(2 \pi^{2}\right)-1}{6 \log \left(2 \pi^{2}\right)-2} \frac{\sqrt{e}}{\pi} \log \left(2 \pi^{2} \Lambda_{S}\right)>0,
$$

and (5) yields (2) with $\rho_{12}=2 \pi^{2}$.

(2) To deal with the cases $n \leq 5$, we use the following values: $J_{1}=2.62205 \cdots$, $J_{2}=\pi / 2, J_{3}=1.19814 \cdots, J_{4}=1$ and $J_{5}=\frac{1}{3} J_{1}=0.87401 \cdots$.

(3) For proving the last assertion of Theorem 1 we use (44) with $S=\{2\}$, and notice that $\Pi_{K}(\{2\}) \geq 1 /\left(1-4^{-n}\right),\left(2 \pi^{2} \Lambda_{S} / \rho_{K}\right)^{n / 2} \leq 4^{-n}, J_{n} \leq J_{2}=\pi / 2$ and

$$
\frac{2 e J_{n}}{\pi^{2}} \frac{n \log \rho_{K}-1}{n \log \rho_{K}-2} \leq \frac{e}{\pi} \frac{2 \log (2700)-1}{2 \log (2700)-2} \leq 1 .
$$




\section{LOWER BOUNDS FOR RELATIVE CLASS NUMBERS}

Recall that a number field $K$ is called a CM-field if $K$ is totally imaginary, hence of even degree $2 n \geq 2$, and if $K$ is a quadratic extension of its maximal totally real subfield $k$. In that situation, the degree of $k$ is equal to $n$, the class number $h_{k}$ of $k$ divides the class number $h_{K}$ of $K$ and we let $h_{K}^{-}=h_{K} / h_{k}$ denote the so-called relative class number of $K$. Recall that

$$
h_{K}^{-}=\frac{Q_{K} w_{K}}{(2 \pi)^{n}} \sqrt{\frac{d_{K}}{d_{k}}} \frac{\kappa_{K}}{\kappa_{k}}
$$

where $w_{K} \geq 2$ and $Q_{K} \in\{1,2\}$ are the number of complex roots of unity in $K$ and the Hasse unit index of $K$, where $d_{K}$ and $d_{k}$ are the absolute values of the discriminants of the number fields $K$ and $k$ and where $\kappa_{K}$ and $\kappa_{k}$ are the residues at $s=1$ of the Dedekind zeta functions of $K$ and $k$ (see Was ). We finally let $\rho_{K}=d_{K}^{1 / 2 n}$ and $\rho_{k}=d_{k}^{1 / n}$ denote the root discriminants of $K$ and $k$, respectively. Hence, $\rho_{k} \leq \rho_{K}$ and $d_{K} / d_{k} \geq d_{K}^{1 / 2}=\rho_{K}^{n}$.

Notation 5. Throughout this paper we adopt the following notation:

$c_{m}=2(\sqrt{m+1}-1)^{2}$.

(In particular, $c_{2}=2(\sqrt{3}-1)^{2}=1.07179 \cdots$ and $c_{3}=2$.)

$\gamma=$ Euler's constant $=0.577215664901 \cdots$

$\kappa_{1}=2+\gamma-\log (4 \pi)=0.046191417392 \cdots$,

$\kappa_{2}=2+\gamma-\log \pi=1.432485779052 \cdots$,

$\kappa_{3}=2+\gamma-\log (\pi / 4)=2.818780140172 \cdots$.

For $n \geq 2$ we set $v_{n}=(n /(n-1))^{n-1} \in[2, e)$.

3.1. Upper bounds for residues of zeta functions. To obtain lower bounds for $h_{K}^{-}$, we will use (6), the lower bounds for $\kappa_{K}$ obtained in Theorem 1 and the following upper bounds for $\kappa_{k}$ :

\section{Proposition 6.}

(1) (See [Lou8, Theorem 1].) Let $k$ be a number field of degree $n>1$. Then

$$
\kappa_{k} \leq\left(\frac{e \log d_{k}}{2(n-1)}\right)^{n-1}=v_{n}\left(\frac{e}{2} \log \rho_{k}\right)^{n-1} .
$$

Moreover, if $\zeta_{k}(\beta)=0$ for some $\beta$ in the range $\frac{1}{2} \leq \beta<1$, then

$$
\kappa_{k} \leq(1-\beta)\left(\frac{e \log d_{k}}{2 n}\right)^{n}=(1-\beta)\left(\frac{e}{2} \log \rho_{k}\right)^{n} .
$$

(2) Let $k$ be a real abelian number field of degree $n>1$ and conductor $f_{k}>1$. Let $X_{k}$ denote the group (of order $n$ ) of primitive Dirichlet characters $\chi$ of conductors $f_{\chi} \geq 1$ associated with this abelian number field $k$ (of degree $n$ ). Set

$$
B_{k}:=\prod_{1 \neq \chi \in X_{k}} \frac{1}{2}\left(\log f_{\chi}+\kappa_{1}\right) \leq \frac{v_{n}}{2^{n-1}}\left(\log \rho_{k}+\kappa_{1}\right)^{n-1} .
$$

Then,

$$
\kappa_{k} \leq B_{k} .
$$

Moreover, if $\zeta_{k}(\beta)=0$ for some $\beta$ in the range $\frac{1}{2} \leq \beta<1$, then

$$
\kappa_{k} \leq \frac{(1-\beta) \log f_{k}}{4} B_{k}
$$


Proof. According to the conductor-discriminant formula, we do have

$$
\begin{aligned}
\prod_{1 \neq \chi \in X_{k}}\left(\log f_{\chi}+\kappa_{1}\right) & \leq\left(\frac{1}{n-1} \log d_{k}+\kappa_{1}\right)^{n-1} \\
& \leq\left(\frac{n}{n-1} \log \rho_{k}+\frac{n}{n-1} \kappa_{1}\right)^{n-1}=v_{n}\left(\log \rho_{k}+\kappa_{1}\right)^{n-1} .
\end{aligned}
$$

Now, $\kappa_{k}=\prod_{1 \neq \chi \in X_{k}} L(1, \chi)=\prod_{1 \neq \chi \in X_{k}}|L(1, \chi)|$. Hence, using Lemma 7 below, we obtain (10) and (11).

Lemma 7. Recall that we set $\kappa_{1}=2+\gamma-\log (4 \pi)=0.046 \cdots$. Let $\chi$ be a primitive even Dirichlet character of conductor $f_{\chi}>1$.

(1) (See [Lou1].) We have1

$$
|L(1, \chi)| \leq\left(\log f_{\chi}+\kappa_{1}\right) / 2 .
$$

(2) (See [Lou6, Corollary 7B] for the quadratic case, and [Lou8 Theorem 7] for the general case). Assume that $L(\beta, \chi)=0$ for some $\beta \in[1 / 2,1)$. Then,

$$
|L(1, \chi)| \leq \frac{1-\beta}{8} \log ^{2} f_{\chi} .
$$

\subsection{On real zeros of Dedekind zeta functions.}

Lemma 8. Set $c_{m}:=2(\sqrt{m+1}-1)^{2}$ (hence, $\frac{1}{3}<c_{1}<1<c_{2}<c_{3}=2$ ).

(1) Let $K$ be a normal CM-field. Set $c=c_{3}=2$ if $K$ is abelian and $c=c_{2}=$ $2(\sqrt{3}-1)^{2}=1.07 \cdots$ otherwise. Then, either (i) there exists some imaginary quadratic subfield $F$ of $K$ such that $\zeta_{F}(s)$ and $\zeta_{K}(s)$ have a common real zero in the range $1-c / \log d_{K} \leq s<1$, or (ii) $\zeta_{k}(s)$ has a real zero in the range $1-c / \log d_{K} \leq s<1$, or (iii) $\zeta_{K}(s) \leq 0$ in the range $1-c / \log d_{K} \leq s<1$.

(2) Let $K$ be a not necessarily normal CM-field such that $\left(\zeta_{K} / \zeta_{k}\right)(s) \geq 0$ for $0<s<12$ Then, either (i) $\zeta_{k}(s)$ has a real zero in the range $1-2 / \log d_{K} \leq s<1$, or (ii) $\zeta_{K}(s) \leq 0$ in the range $1-2 / \log d_{K} \leq s<1$.

(3) Let $N$ denote the normal closure of a $C M$-field $K$. Then, either (i) there exists some imaginary quadratic subfield $F$ of $K$ such that $\zeta_{F}(s)$ and $\zeta_{K}(s)$ have a common real zero in the range $1-\left(c_{1} / \log d_{N}\right) \leq s<1$, or (ii) $\zeta_{k}(s)$ has a real zero in the range $1-\left(c_{1} / \log d_{N}\right) \leq s<1$, or (iii) $\zeta_{K}(s) \leq 0$ in the range $1-\left(c_{1} / \log d_{N}\right) \leq s<1$.

(4) Let $N$ be the normal closure of a number field $K$ of degree $m$. Then, $d_{N}$ divides $d_{K}^{[N: \mathbf{Q}]}$ and the degree $[N: \mathbf{Q}]$ of $N$ divides $m$ !.

(5) Let $F$ be an imaginary quadratic number field. Then, the Dedekind zeta function $\zeta_{F}(s)$ of $F$ has no real zero in the range $1-\left(6 / \pi \sqrt{d_{F}}\right) \leq s<1$.

(6) For any $\epsilon>0$ there exists an ineffective constant $c_{\epsilon}>0$ such that the Dedekind zeta functions $\zeta_{F}(s)$ of the imaginary quadratic number fields $F$ have no real zero in the range $1-\left(c_{\epsilon} / d_{F}^{\epsilon / 2}\right) \leq s<1$.

Proof. Let $m \geq 1$ be a given positive integer and $K$ be a number field of discriminant $d_{K}>\exp (2(\sqrt{m+1}-1))$. Then, its Dedekind zeta function $\zeta_{K}(s)$ has at most $m$ real zeros in the range $1-\left(c_{m} / \log d_{K}\right) \leq s<1$ (this result is a generalisation of [Sta3, Lemma 3] and its proof is given in [LLO, Lemma 15]).

\footnotetext{
${ }^{1}$ We could choose $\kappa_{1}=0$, by [Ram Corollary 1].

${ }^{2}$ For example, $K$ is a dihedral or quaternion CM-field of degree $2 n \geq 8$.
} 
(1) The abelian case is easy to deal with by using the factorization of $\zeta_{K}(s)$ as a product of Dirichlet $L$-series. Let us now deal with the normal case. Assume that we are neither in case (i) nor in case (iii). Since we are not in case (iii), there exists $s_{1}$ in the range $1-c_{2} / \log d_{K} \leq s<1$ such that $\zeta_{K}\left(s_{1}\right)>0$. Since $\kappa_{K}$ is positive and since $\zeta_{K}(s)$ has a simple pole at $s=1$, it follows that $\lim _{s \uparrow 1} \zeta_{K}(s)=-\infty$. Hence, $\zeta_{K}(s)$ has a real zero $\beta$ of odd multiplicity $n_{\beta} \geq 1$ in the range $1-c_{2} / \log d_{K} \leq s_{1} \leq s<1$. However, in this range we have $n_{\beta} \leq 2$. Hence, $n_{\beta}=1$. According to Sta3. Theorem 3], there exists some quadratic subfield $F$ of $K$ such that for $E \subseteq K$ we have $\zeta_{E}(\beta)=0$ if and only if $F \subseteq E$. In particular, $\zeta_{F}(\beta)=0$. Since we are not in case (i), we obtain that $F$ is real. Hence, $F \subseteq k$, which implies $\zeta_{k}(\beta)=0$, and we are in case (ii).

(2) Easy.

(3) Assume that we are neither in case (i) nor in case (iii). Since we are not in case (iii), there exists $s_{1}$ in the range $1-\left(c_{1} / \log d_{K}\right) \leq s<1$ such that $\zeta_{K}\left(s_{1}\right)>0$. Since $\lim _{s \uparrow 1} \zeta_{K}(s)=-\infty$, there exists some real zero $\beta$ of $\zeta_{K}$ in the range $s_{1} \leq s<1$. Since $N / K$ is normal, $\zeta_{K}$ divides $\zeta_{N}$ (see [FM], Corollary 2] for a short proof of the Aramata-Brauer Theorem). Hence, $\zeta_{N}(\beta)=0$ and $\beta$ is a simple zero of $\zeta_{N}$, for $1-\left(c_{1} / \log d_{N}\right) \leq s_{1} \leq \beta<1$. According to Sta3, Theorem 3], there exists a quadratic subfield $F \subseteq N$ such that $E \subseteq N$ and $\zeta_{E}(\beta)=0$ if and only if $F \subseteq E$. In particular, $F \subseteq K$ (for $\zeta_{K}(\beta)=0$ ) and $\zeta_{F}(\beta)=0$. Since we are not in case (i), then $F$ is real. Hence $F \subseteq k$ and $\zeta_{k}(\beta)=0$ and we are in case (ii).

(4) Use [Sta3, Lemma 7].

(5) See [Bes] (the proof of this result was announced to appear in Hof, Reference 2] but it has in fact never been published yet).

(6) See [Pin1, Siegel's Theorem II], [Pin2, Theorem 1] and [Sie.

3.3. Lower bounds for relative class numbers. We are now in a position to obtain lower bounds for relative class numbers.

Theorem 9. Let $K$ be a normal $C M$-field of degree $2 n>2$ and root discriminant $\rho_{K} \geq 50$. Assume that $K$ contains no imaginary quadratic subfield, or that the Dedekind zeta functions of the imaginary quadratic subfields of $K$ have no real zero in the range $1-\left(c / \log d_{K}\right) \leq s<1$.

(1) Set $c=c_{2}=2(\sqrt{3}-1)^{2}$. We have

$$
h_{K}^{-} \geq \frac{c Q_{K} w_{K} \sqrt{d_{K} / d_{k}}}{2 \pi e^{c / 2} v_{n}\left(\pi e \log \rho_{k}\right)^{n-1} \log d_{K}} \geq \frac{c}{2 n v_{n} e^{c / 2-1}}\left(\frac{\sqrt{\rho_{K}}}{\pi e \log \rho_{K}}\right)^{n}
$$

and for each entry $2 m$ in Table 2 below, $2 n \geq 2 m$ and $\rho_{K} \geq \rho_{2 m}$ imply $h_{K}^{-}>1$.

(2) Moreover, assume that $k$ is abelian and set $c=c_{3}=2$ if $K$ is abelian, and set $c=c_{2}=2(\sqrt{3}-1)^{2}$ otherwise. Then, we have the better lower bound

$$
h_{K}^{-} \geq \frac{c Q_{K} w_{K} \sqrt{d_{K} / d_{k}}}{e^{c / 2}(2 \pi)^{n} B_{k} \log d_{K}} \geq \frac{c}{2 n v_{n} e^{c / 2}}\left(\frac{\sqrt{\rho_{K}}}{\pi\left(\log \rho_{K}+\kappa_{1}\right)}\right)^{n}
$$

(recall that we have set $\left.\kappa_{1}=2+\gamma-\log (4 \pi)=0.046 \cdots\right)$, and for each entry $2 m$ in Table 3 below, $2 n \geq 2 m$ and $\rho_{K} \geq \rho_{2 m}$ imply $h_{K}^{-}>1$. 
Table $2: c=c_{2}$

\begin{tabular}{|c|c|c|c|c|c|c|c|c|c|}
\hline $\begin{array}{c}2 m \\
\rho_{2 m}\end{array}$ & $\begin{array}{c}4 \\
38100\end{array}$ & $\begin{array}{c}6 \\
31000\end{array}$ & $\begin{array}{c}8 \\
25000\end{array}$ & $\begin{array}{c}10 \\
21000\end{array}$ & $\begin{array}{c}20 \\
13000\end{array}$ & $\begin{array}{c}40 \\
9200\end{array}$ & $\begin{array}{c}100 \\
7000\end{array}$ & $\begin{array}{c}200 \\
6260\end{array}$ & $\begin{array}{c}\infty \\
5383\end{array}$ \\
\hline \multicolumn{10}{|c|}{ Table 2 (continued) $: c=2$} \\
\hline $2 m$ & 4 & 6 & 8 & 10 & 20 & 40 & 100 & 200 & $\infty$ \\
\hline$\rho_{2 m}$ & 31300 & 27200 & 22600 & 19400 & 12500 & 9000 & 7000 & 6230 & 5383 \\
\hline
\end{tabular}

Table $3: k$ is abelian and $c=c_{2}$

\begin{tabular}{|c|ccccccccc|}
\hline $2 m$ & 4 & 6 & 8 & 10 & 20 & 40 & 100 & 200 & $\infty$ \\
$\rho_{2 m}$ & 11100 & 5800 & 3710 & 2700 & 1220 & 726 & 490 & 418 & 342 \\
\hline
\end{tabular}

Table 3 (continued) $: k$ is abelian and $c=2$

\begin{tabular}{|c|ccccccccc|}
\hline $2 m$ & 4 & 6 & 8 & 10 & 20 & 40 & 100 & 200 & $\infty$ \\
$\rho_{2 m}$ & 9000 & 5100 & 3340 & 2480 & 1170 & 709 & 486 & 416 & 342 \\
\hline
\end{tabular}

Proof. Let us first prove (13). According to Point 1 of Lemma 8 , there are two cases to consider.

(1) Assume that $\zeta_{k}$ has no real zero in the range $1-c / \log d_{K} \leq s<1$. Then $\zeta_{K}\left(1-\left(c / \log d_{K}\right)\right) \leq 0$ and using (2) with $S=\emptyset$, we obtain

$$
\kappa_{K} \geq \frac{c}{e^{c / 2} \log d_{K}}
$$

Using (10) we conclude that

$$
\frac{\kappa_{K}}{\kappa_{k}} \geq \frac{c}{e^{c / 2} B_{k} \log d_{K}}
$$

(2) Assume that $\zeta_{k}(\beta)=0$ for some $\beta \in\left[1-c / \log d_{K}, 1\right)$. Then $\zeta_{K}(\beta)=0 \leq 0$ and using (11) with $S=\emptyset$, we obtain

$$
\kappa_{K} \geq \frac{1-\beta}{2 e^{c / 2}}
$$

Using (11) we conclude that

$$
\frac{\kappa_{K}}{\kappa_{k}} \geq \frac{2}{e^{c / 2} B_{k} \log f_{k}} .
$$

Since $d_{K}>d_{k} \geq f_{k}$ and since $c \leq 2$, the right-hand side of (15) is greater than or equal to the right-hand side of (14), and (14) is always valid.

Using (14), (9) and (6)), we obtain the first lower bound in (13). To deduce the second one, we use $\log d_{K}=2 n \log \rho_{K}, \sqrt{d_{K} / d_{k}} \geq d_{K}^{1 / 4}=\rho_{K}^{n / 2}$ and $\rho_{k} \leq \rho_{K}$.

To prove (12), we use (7) and (8), instead of (10) and (11).

Remarks 10. According to (4), we could easily improve upon (11). For example, we have: let $K$ be a totally imaginary number field of degree $2 n \geq 4$ and root discriminant $\rho_{K}:=d_{K}^{1 / 2 n} \geq 98$. Assume that $\zeta_{K}(\beta) \leq 0$ for some $\beta \in[1-$ $\left.\left(2 / \log d_{K}\right), 1\right)$. Then,

$$
\kappa_{K} \geq \frac{4}{5}(1-\beta) e^{(\beta-1) / 2} .
$$

The reader can easily check that by following the proof of Theorem 9 and by using (3), or (16) with $S=\emptyset$, we can slightly improve upon [LPP, Proposition 4.2].

In the same way, by using Point 2 of Lemma 8 we obtain: 
Theorem 11. Let $K$ be a not necessarily normal $C M$-field of degree $2 n \geq 2$ such that $\left(\zeta_{K} / \zeta_{k}\right)(s) \geq 0$ for $0<s<1$ 年 Then, (12) holds with $c=2$. In particular, for each entry $2 m$ in Table 2 , we have $h_{K}^{-}>1$ as soon as $2 n \geq 2 m$ and $\rho_{K} \geq \rho_{2 m}$. Moreover, if $k$ is abelian 4 then (13) holds with $c=2$. In particular, for each entry $2 m$ in Table 3, we have $h_{K}^{-}>1$ as soon as $n \geq m$ and $\rho_{K} \geq \rho_{2 m}$.

Finally, by using (3), (7), (8) and Points 3 and 4 of Lemma 8 we obtain:

Theorem 12. Set $c=c_{1}=2(\sqrt{2}-1)^{2}$. Let $K$ be a not necessarily normal CM-field of degree $2 n>2$ and root discriminant $\rho_{K} \geq 2800$, let $m_{N}:=[N: \mathbf{Q}]$ denote the degree of its normal closure $N$ and assume that $K$ contains no imaginary quadratic subfield or that the real zeros in the range $1-\left(c / \log d_{N}\right) \leq s<1$ of the Dedekind zeta functions of the imaginary quadratic subfields of $K$ are not zeros of $\zeta_{K}(s)$. Then,

$$
h_{K}^{-} \geq \frac{c Q_{K} w_{K} \sqrt{d_{K} / d_{k}}}{4 n m_{N} e^{c / 2}\left(\pi e \log \rho_{K}\right)^{n}} .
$$

When dealing with small class number problems for CM-fields $K$, one can assume that either $K$ contains no imaginary quadratic subfield or that $\zeta_{F}(s)<0$ in the range $0<s<1$ for all the imaginary quadratic subfields $F$ of $K$, which enables one to use Theorems 9 and 12. Indeed, the class number of any imaginary quadratic subfield of $K$ divides $4 h_{K}^{-}$(see Oka]), all the imaginary quadratic fields of small class numbers are known (e.g. those of class numbers dividing 4 were determined in [Arn], Bak1], Bak2], MW], Sta1] and [Sta2]), and one can easily check numerically that $\zeta_{F}(s)<0,0<s<1$, for these few imaginary quadratic fields $F$. However, in order to prove in Section 4 a Brauer-Siegel-like result for relative class numbers of CM-fields, we prove:

Theorem 13. Let $\gg_{\epsilon}$ mean that the constants involved in the considered lower bound depend on $\epsilon$ only. Let $K$ be a not necessarily normal CM-field of degree $2 n>4$ and root number $\rho_{K} \geq 2800$. Assume that $K$ contains an imaginary quadratic subfield $F$ and that $\zeta_{F}(\beta)=\zeta_{K}(\beta)=0$ for some $\beta \in\left[-\left(2 / \log d_{K}\right), 1\right)$. Then,

$$
h_{K}^{-} \geq \frac{6}{\pi^{2} e^{2}} \frac{\left(d_{K} / d_{k}\right)^{\frac{1}{2}-\frac{1}{n}}}{\left(\pi e \log \rho_{K}\right)^{n-1}} \geq \frac{6}{\pi^{2} e^{2} \sqrt{\rho_{K}}}\left(\frac{\sqrt{\rho_{K}}}{\pi e \log \rho_{K}}\right)^{n-1}
$$

and (ineffectively)

$$
h_{K}^{-} \gg_{\epsilon} \frac{\left(d_{K} / d_{k}\right)^{\frac{1}{2}-\frac{\epsilon}{n}}}{\left(\pi e \log \rho_{K}\right)^{n-1}},
$$

and for each entry $2 m$ in Table 4 below, $2 n \geq 2 m$ and $\rho_{K} \geq \rho_{2 m}$ imply $h_{K}^{-}>1$.

Table 4: $c=c_{2}$

\begin{tabular}{|c|ccccccccc|}
\hline $2 m$ & 6 & 8 & 10 & 12 & 20 & 40 & 100 & 200 & $\infty$ \\
$\rho_{2 m}$ & $5 \cdot 10^{11}$ & $5 \cdot 10^{7}$ & $3 \cdot 10^{6}$ & $5 \cdot 10^{5}$ & 50000 & 15000 & 7800 & 6500 & 5383 \\
\hline
\end{tabular}

According to (12) and (18), it follows that $h_{K}^{-} \rightarrow \infty$ as $[K: \mathbf{Q}]=2 n \rightarrow \infty$ for normal CM-fields of root discriminants $\rho_{K}=d_{K}^{1 / 2 n} \geq c_{\infty}:=5400$.

\footnotetext{
${ }^{3}$ For example, $K$ is a dihedral CM-field of degree $2 n \geq 8$ as in [LO].

${ }^{4}$ For example, $K$ is a quaternion or a dihedral octic CM-field as in Lou3] and [Lou5, Section 2.3.1].
} 
Proof. According to Point 4 of Lemma 8 we have $1-\beta \geq \frac{6}{\pi}\left(d_{K} / d_{k}\right)^{-1 / n}$ and $1-\beta \gg_{\epsilon}\left(d_{K} / d_{k}\right)^{-\epsilon / n}$ (notice that $\left.\sqrt{d_{F}}=\rho_{F} \leq \rho_{K} \leq\left(d_{K} / d_{k}\right)^{1 / n}\right)$. According to (3)), we have $\kappa_{K} \geq(1-\beta) / e$, and we obtain

$$
\kappa_{K} \geq \frac{6}{\pi e}\left(d_{K} / d_{k}\right)^{-1 / n} \text { and } \kappa_{K} \gg_{\epsilon}\left(d_{K} / d_{k}\right)^{-\epsilon / n} .
$$

Using (6), (7) and (20), we obtain (18) and (19).

\section{A Brauer-Siegel-like Result on the asymptotic Behavior of RELATIVE CLASS NUMBERS OF CM-FIELDS}

By using our previous lower bounds for relative class numbers of CM-fields (see Theorems 9, 12 and 13), we now prove Brauer-Siegel-like results about the asymptotic behavior of relative class numbers of CM-fields. In [HJ p. 554] it is said that the restriction $\rho_{K} \rightarrow \infty$ precludes one from deducing from the BrauerSiegel theorem that there exists some sufficiently large constant $C>0$ such that $h_{K} \rightarrow \infty$ as $[K: \mathbf{Q}]=2 n \rightarrow \infty$ for normal CM-fields $K$ of root discriminants $\rho_{K}=d_{K}^{1 / 2 n} \geq C$. The Brauer-Siegel-like results we will obtain here prove that we may choose $C=5400$. In [Mur2, Proposition 4.1] it is said that as $K$ ranges over the set of CM-fields of degrees $2 n \leq 8$ and $2 n \neq 4$ we have $h_{K} \rightarrow \infty$ effectively. The Brauer-Siegel-like results we will obtain here prove that for any given $B$ we have $h_{K} \rightarrow \infty$ effectively as $K$ ranges over the set of CM-fields of degrees $2 n \leq B$. The Brauer-Siegel-like results for relative class number of CM-fields we are going to prove (and which generalize those we obtained in Lou4 for imaginary abelian number fields) are as follows:

\section{Theorem 14.}

(1) Let $K$ range over a sequence of normal CM-fields such that their root discriminants $\rho_{K}$ tend to infinity (e.g. let $K$ range over a sequence of imaginary abelian number fields 5), or let $K$ range over a sequence of not necessarily normal $C M$-fields of a given degree. Let o(1) denote an error term that tends to zero as $\rho_{K}$ goes to infinity.

We have

$$
\left(\frac{1}{2}+o(1)\right) \log \left(d_{K} / d_{k}\right) \geq \log h_{K}^{-} \geq\left(\frac{1}{2}+o(1)\right) \log \left(d_{K} / d_{k}\right)
$$

i.e., $\log h_{K}^{-}$is asymptotic to $\frac{1}{2} \log \left(d_{K} / d_{k}\right)$, which implies that

$$
h_{K}^{-} \gg d_{K}^{\frac{1}{4}+o(1)} \text {. }
$$

The upper bound on $\log h_{K}^{-}$in (21) is effective and explicit.

If $K$ contains no imaginary quadratic subfield, then the lower bounds for $\log h_{K}^{-}$ in (21) and (22) are effective and explicit.

If $K$ contains an imaginary quadratic subfield, then the lower bounds for $h_{K}^{-}$ in (21) and (22) are not effective, but we have the following effective and explicit weaker lower bound:

$$
\log h_{K}^{-} \geq\left(\frac{1}{2}-\frac{1}{n}+o(1)\right) \log \left(d_{K} / d_{k}\right)
$$

\footnotetext{
${ }^{5}$ For in that case it follows that $\rho_{K} \geq \sqrt{f_{K}}$ (see [Mur1 Corollary 1]).
} 
which implies the following effective and explicit lower bound:

$$
h_{K}^{-} \gg d_{K}^{\frac{1}{4}-\frac{1}{2 n}+o(1)} .
$$

Finally, in the situations where the error terms o(1) in (21), (221), (23) and (24) are declared to be effective and explicit, they are of the type oo 1$)=O\left(\left(\log \log \rho_{K}\right) /\right.$ $\left.\log \rho_{K}\right)$.

(2) If $K$ ranges over not necessarily normal CM-fields of a given degree, then $h_{K}^{-} \longrightarrow \infty$ effectively and explicitly as $d_{K} \longrightarrow \infty$.

For any given $h \geq 1$ there exists $\rho_{h}$ effective such that $h_{K}^{-}>h$ for all normal $C M$-fields $K$ of root discriminants $\rho_{K} \geq \rho_{h}$.

In particular, $h_{K}^{-}>1$ for all normal CM-fields $K$ of root discriminants $\rho_{K} \geq$ $\rho_{1}=40000$.

Moreover, $h_{K} \rightarrow \infty$ as $[K: \mathbf{Q}]=2 n \rightarrow \infty$ for normal CM-fields $K$ of root discriminants $\rho_{K}=d_{K}^{1 / 2 n} \geq C=5400$.

\subsection{Proof of Theorem 14 .}

Lemma 15. Let $K$ be a CM-field of degree $2 n$. Then,

$$
\log h_{K}^{-} \leq\left(\frac{1}{2}+o(1)\right) \log \left(d_{K} / d_{k}\right)
$$

where o $(1)=O\left(\left(\log \log \rho_{K}\right) / \log \rho_{K}\right)$ is an explicit error term that tends to zero as $\rho_{K}$ goes to infinity.

Proof. Since $\phi(w) \geq \sqrt{w / 2}$ for $w \geq 2$ and since $\phi\left(w_{K}\right)$ must divide $2 n$, we have $w_{K} \leq 8 n^{2}$. Moreover, $d_{K} / d_{k} \leq d_{K}=\rho_{K}^{2 n}$. Hence, using [Lou7, Corollary 3], we obtain

$$
h_{K}^{-} \leq 2 Q_{K} w_{K} \sqrt{d_{K} / d_{k}}\left(\frac{e}{4 \pi n} \log \left(d_{K} / d_{k}\right)\right)^{n} \leq 32 n^{2} \sqrt{d_{K} / d_{k}}\left(\frac{e}{2 \pi} \log \rho_{K}\right)^{n}
$$

and the desired result, by using $\log \left(d_{K} / d_{k}\right) \geq \log \left(d_{K}^{1 / 4}\right)=n \log \rho_{K}$.

1. The first point of Theorem 14 follows from Lemma 15 and Theorems 912 and 13 (to prove the last assertion of the first point of Theorem 14, recall that $\left.\log \left(d_{K} / d_{k}\right) \geq \log \left(d_{K}^{1 / 2}\right)=n \log \rho_{K}\right)$.

2. The first and second assertions of the second point of Theorem 14 follow from the first point of Theorem 14 (for CM-fields of degrees $2 n>4$ ) and from the following known results (for CM-fields of degrees $2 n \leq 4$ ):

\section{Lemma 16.}

(1) (See Oes].) For every $\epsilon>0$ we have an effective and explicit lower bound $h_{F}^{-} \gg_{\epsilon} \log ^{1-\epsilon} d_{F}$ for the class numbers $h_{F}$ of the imaginary quadratic fields $F$.

(2) If $K=F_{1} F_{2}$ is an imaginary bicyclic biquadratic field (where $F_{1}$ and $F_{2}$ denote the two imaginary quadratic subfields of $K)$, then $d_{K} / d_{k}=d_{F_{1}} d_{F_{2}}$ and

$$
h_{K}^{-}=\frac{Q_{K}}{2} h_{F_{1}} h_{F_{2}} .
$$

Hence, we have an effective and explicit lower bound $h_{K}^{-} \gg_{\epsilon} \log ^{1-\epsilon} d_{K}$.

(3) If $K$ is a non-normal quartic CM-field, then its normal closure $N$ is a dihedral octic CM-field, $d_{N} / d_{N^{+}}=\left(d_{K} / d_{k}\right)^{2}$, and

$$
h_{N}^{-}=\frac{Q_{N}}{2}\left(h_{K}^{-}\right)^{2} .
$$


Therefore, $\log h_{K}^{-}$is effectively and explicitly asymptotic to $\frac{1}{2} \log \left(d_{K} / d_{k}\right)$.

3. The third assertion of the second point of Theorem 14 follows from the fact that if $h_{K}^{-}=1$ and $K$ contains an imaginary quadratic field $F$, then $h_{F}$ divides 4 (see [Oka]). Hence $F$ is known (see [Arn], Bak1, Bak2], [MW], [Sta1] and [Sta2]), and numerical computations easily yield that $\zeta_{F}(s)<0$ for these few imaginary quadratic fields $F$. Hence, the first point of Theorem 9 yields that $h_{K}^{-}>1$ for all normal CM-fields $K$ of root discriminants $\rho_{K}>40000$. (We could also use Theorem 13 and the solution of the class number one problem for the imaginary quadratic fields (see [Bak1] and Sta1]) and for the imaginary biquadratic bicyclic fields (see $[\overline{\mathrm{BP}}]$ ), but we would obtain the weaker following result: $h_{K}^{-}>1$ for all normal CM-fields $K$ of root discriminants $\rho_{K}>7 \cdot 10^{11}$.)

4. Finally, the fourth assertion of the second point of Theorem 14 follows from the last assertion of Theorem 13

Remarks 17. It is possible to deduce from the usual Brauer-Siegel theorem for class numbers of number fields the following Brauer-Siegel-like result for relative class numbers of normal CM-fields, which improves upon [HH] Lemma 4] (which is given only for CM-fields of a given degree) but is less satisfactory than our previous Theorem[14 (for it is ineffective in the case that $N$ contains no imaginary quadratic subfield):

Theorem 18. If $N$ ranges over a sequence of normal CM-fields such that their root discriminants $\rho_{N}$ tend to infinity, then we have

$$
\log h_{N}^{-} \sim \frac{1}{2} \log \left(d_{N} / d_{N^{+}}\right),
$$

which implies

$$
h_{N}^{-} \gg d_{N}^{\frac{1}{4}+o(1)}
$$

where $o(1)$ is an error term that tends to zero as $\rho_{N}$ goes to infinity.

\section{BETTER LOWER BOUNDS FOR RELATIVE ClASS NUMBERS}

The aim of this section is to improve upon, in the case that $k$ is abelian, the explicit lower bounds for relative class numbers of CM-fields $K$ that we obtained in the previous section. To this end, we choose $S=\{2\}$ and use Theorem 1 to get better lower bounds (depending on the behavior of 2 in $K$ ) for the term $\kappa_{K}$ in (6). Moreover, using the results of [Lou9] we will be able to get better upper bounds (depending on the behavior of 2 in $k$ ) for the term $\kappa_{k}$ in (6). Putting everything together, we will obtain Theorem 22, which improves upon the lower bounds for relative class numbers that we obtained in Theorem 9

5.1. Upper bounds for $|L(1, \chi)|$.

Lemma 19. Let $\chi$ be a primitive even Dirichlet character of conductor $f_{\chi}>1$.

(1) (See [Lou1] and [Lou9].) Set 6

$$
\kappa_{\chi}:= \begin{cases}\kappa_{1}=2+\gamma-\log (4 \pi)=0.046 \cdots & \text { if } \chi(2)=+1, \\ \kappa_{2}=2+\gamma-\log \pi=1.432 \cdots & \text { if } \chi(2)=0, \\ \kappa_{3}=2+\gamma-\log (\pi / 4)=2.818 \cdots & \text { if } \chi(2) \neq 0,+1 .\end{cases}
$$

${ }^{6}$ We could choose $\kappa_{1}=0$ and $\kappa_{2}=\log 4=1.386 \cdots$, by Ram, Corollaries 1 and 2]. 
We have

$$
|L(1, \chi)| \leq \frac{1}{4}\left|1-\frac{\chi(2)}{2}\right|^{-1}\left(\log f_{\chi}+\kappa_{\chi}\right) .
$$

(2) (See [Lou6, Corollary 7B] for the quadratic case and [Lou8, Theorem 7] for the general case.) If $L(\beta, \chi)=0$ for some $\beta \in[1 / 2,1)$, then

$$
|L(1, \chi)| \leq \frac{1-\beta}{8} \log ^{2} f_{\chi} .
$$

\subsection{Upper bounds for residues of zeta functions.}

Proposition 20. Let $k$ be a real abelian number field of degree $n>1$ and conductor $f_{k}>1$. Let $X_{k}$ denote the group (of order $n$ ) of primitive Dirichlet characters $\chi$ of conductors $f_{\chi} \geq 1$ associated with this abelian number field $k$ (of degree $n$ ). Let $e, f$ and $g=n /(e f)$ denote the index of ramification of 2 in $k$, the inertia degree of 2 in $k$ and the number of prime ideals of $k$ above 2 , respectively. (Hence, $\left.\Pi_{k}(\{2\})=\left(1-2^{-f}\right)^{-g}.\right)$ Set

$$
\begin{aligned}
\kappa_{k} & :=\frac{1}{n} \sum_{1 \neq \chi \in X_{k}} \kappa_{\chi}\left(\text { with } \kappa_{\chi}\right. \text { as in (26)) } \\
& \leq \kappa_{n, f, g}:=\frac{(g-1) \kappa_{1}+(n-f g) \kappa_{2}+(f g-g) \kappa_{3}}{n}
\end{aligned}
$$

(hence $0<\kappa_{k} \leq \kappa_{n, f, g} \leq \kappa_{3} \leq 3$ ) and

$$
B_{k}(\{2\}):=\frac{1}{2} \prod_{1 \neq \chi \in X_{k}} \frac{1}{4}\left(\log f_{\chi}+\kappa_{\chi}\right) \leq \frac{v_{n}}{2 \cdot 4^{n-1}}\left(\log \rho_{k}+\kappa_{k}\right)^{n-1} .
$$

Then,

$$
\kappa_{k} \leq \Pi_{k}(\{2\}) B_{k}(\{2\}) .
$$

Moreover, if $\zeta_{k}(\beta)=0$ for some $\beta \in[1 / 2,1)$, then

$$
\kappa_{k} \leq \frac{3(1-\beta) \log f_{k}}{4} \Pi_{k}(\{2\}) B_{k}(\{2\}) .
$$

Proof. To deduce (30) from (29), we notice that, according to [Was, Theorem 3.7],

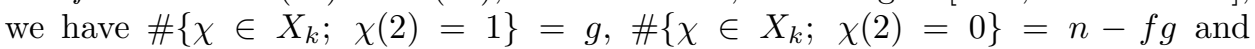
$\#\left\{\chi \in X_{k} ; \chi(2) \neq 0,1\right\}=n-g-(n-f g)=f g-g$. Using the fact that the geometric mean is less than or equal to the arithmetic mean and the conductordiscriminant formula $\prod_{1 \neq \chi \in X_{k}} f_{\chi}=d_{k}=\rho_{k}^{n}$, we do have

$$
B_{k}(\{2\}) \leq \frac{1}{2 \cdot 4^{n-1}}\left(\frac{1}{n-1} \sum_{1 \neq \chi \in X_{k}}\left(\log f_{\chi}+\kappa_{\chi}\right)\right)^{n-1}=\frac{v_{n}}{2 \cdot 4^{n-1}}\left(\log \rho_{k}+\kappa_{k}\right)^{n-1} .
$$

Noticing that

$$
\prod_{1 \neq \chi \in X_{k}}\left(1-\frac{\chi(2)}{2}\right)^{-1}=\frac{1}{2} \Pi_{k}(\{2\})
$$

and using (27) for all the $1 \neq \chi \in X_{k}$, we obtain

$$
\kappa_{k}=\prod_{1 \neq \chi \in X_{k}}|L(1, \chi)| \leq \frac{\prod_{k}(\{2\})}{2 \cdot 4^{n-1}} \prod_{1 \neq \chi \in X_{k}}\left(\log f_{\chi}+\kappa_{\chi}\right),
$$


which proves (32). Now, if $\zeta_{k}(\beta)=0$, then $L\left(\beta, \chi_{0}\right)=0$ for some $1 \neq \chi_{0} \in X_{k}$. Using (28) we obtain

$$
\begin{aligned}
\left|L\left(1, \chi_{0}\right)\right| \leq \frac{1-\beta}{8} \log ^{2} f_{\chi_{0}} & \leq \frac{1-\beta}{8} \cdot \frac{3}{2}\left|1-\frac{\chi_{0}(2)}{2}\right|^{-1} \cdot \log ^{2} f_{\chi_{0}} \\
& \leq \frac{3(1-\beta) \log f_{k}}{4}\left|1-\frac{\chi_{0}(2)}{2}\right|^{-1} \frac{\log f_{\chi_{0}}+\kappa_{\chi_{0}}}{4},
\end{aligned}
$$

which, in using (27) for all the $\chi \in X_{k} \backslash\left\{1, \chi_{0}\right\}$, yields (33).

Remarks 21. Notice that in the special case that the prime 2 is inert in the real abelian number field $k$ of degree $n$, then (31) and (32) yield

$$
\kappa_{k} \leq \frac{v_{n}}{2^{n-1}\left(2^{n}-1\right)}\left(\log \rho_{k}+\kappa_{3}\right)^{n-1},
$$

whereas (9) and (10) only yield $\kappa_{k} \leq v_{n}\left(\log \rho_{k}+\kappa_{1}\right)^{n-1} / 2^{n-1}$.

\subsection{Lower bounds for relative class numbers.}

Theorem 22. Let $K$ be a normal $C M$-field of degree $2 n \geq 2 m>2$ and root discriminant $\rho_{K} \geq \rho_{2 m,\{2\}}$ with $\rho_{2 m,\{2\}}$ as in Table 1. Assume that $k$ is abelian. Set $c=c_{3}=2$ if $K$ is abelian, and set $c=c_{2}=2(\sqrt{3}-1)^{2}$ otherwise. Assume that $K$ contains no imaginary quadratic subfield or that the Dedekind zeta functions of the imaginary quadratic subfields of $K$ have no real zero in the range $1-\left(c / \log d_{K}\right) \leq$ $s<1$. Then,

$$
h_{K}^{-} \geq \frac{c}{e^{c / 2}} \frac{Q_{K} w_{K} \Pi_{K / k}(\{2\}) \sqrt{d_{K} / d_{k}}}{(2 \pi)^{n} B_{k}(\{2\}) \log d_{K}}
$$

with $B_{k}(\{2\})$ as in (31) and $\Pi_{K / k}(\{2\})=\Pi_{K}(\{2\}) / \Pi_{k}(\{2\})$.

Therefore, setting $C_{n, f, g}=2\left(1+2^{-f}\right)^{-g / n} \in[4 / 3,2)$, we have

$$
h_{K}^{-} \geq \frac{c}{2 n v_{n} e^{c / 2}}\left(\frac{C_{n, f, g} \sqrt{\rho_{K}}}{\pi\left(\log \rho_{K}+\kappa_{n, f, g}\right)}\right)^{n}
$$

(with $f, g$ and $\kappa_{n, f, g}$ as in Proposition 20). In particular, for each entry $2 n$ in Table 6 below, we have $h_{K}^{-}>1$ as soon as $\rho_{K} \geq \rho_{2 n}$.

Table 6 (compare with Table 3 )

\begin{tabular}{|c|cccccccc|}
\hline $2 n$ & 4 & 6 & 8 & 10 & 20 & 40 & 100 & 200 \\
$\rho_{2 n}$ for $c=c_{2}$ & 5217 & 2704 & 1707 & 1228 & 538 & 310 & 206 & 181 \\
$\rho_{2 n}$ for $c=2$ & 4233 & 2344 & 1530 & 1124 & 513 & 303 & 205 & 180 \\
\hline
\end{tabular}

Proof. According to Points 1 and 2 of Lemma 8 there are two cases to consider.

(1) Assume that $\zeta_{k}$ has no real zero in the range $1-c / \log d_{K} \leq s<1$. Then $\zeta_{K}\left(1-\left(c / \log d_{K}\right)\right) \leq 0$ and using (2) with $S=\{2\}$, we obtain

$$
\kappa_{K} \geq \frac{c \Pi_{K}(\{2\})}{e^{c / 2} \log d_{K}} .
$$

Using (32) we conclude that

$$
\frac{\kappa_{K}}{\kappa_{k}} \geq \frac{c \Pi_{K / k}(\{2\})}{e^{c / 2} B_{k}(\{2\}) \log d_{K}} .
$$


(2) Assume that $\zeta_{k}(\beta)=0$ for some $\beta \in\left[1-\left(c / \log d_{K}\right), 1\right)$. Then $\zeta_{K}(\beta)=0 \leq 0$ and using (11) with $S=\{2\}$, we obtain

$$
\kappa_{K} \geq \frac{(1-\beta) \Pi_{K / k}(\{2\})}{2 e^{c / 2}} .
$$

Using (33) we conclude that

$$
\frac{\kappa_{K}}{\kappa_{k}} \geq \frac{c \Pi_{K / k}(\{2\})}{e^{c / 2} B_{k}(\{2\}) \frac{3 c}{2} \log f_{k}} .
$$

Now, if $n \geq 3$, then $d_{K} \geq d_{k}^{2} \geq f_{k}^{3}$ (see [Mur1, Corollary 1]), and if $n=2$, then $K$ is cyclic quartic and here again $d_{K}=f_{K}^{2} f_{k} \geq f_{k}^{3}$. Hence, we always have

$$
\frac{3 c}{2} \log f_{k} \leq 3 \log f_{k} \leq \log d_{K}
$$

(for $c \leq 2$ ). Therefore, the right-hand side of (37) is greater than or equal to the right-hand side of (36), and (36) is always valid. Using (36), (31) and (6), we obtain (34). To deduce (35), we use $\log d_{K}=2 n \log \rho_{K}, \sqrt{d_{K} / d_{k}} \geq d_{K}^{1 / 4}=\rho_{K}^{n / 2}, \rho_{k} \leq \rho_{K}$ and

$$
2^{n} \Pi_{K / k}(\{2\})=2^{n}\left(1-\epsilon_{2} / 2^{f}\right)^{-g} \geq\left(C_{n, f, g}\right)^{n}
$$

(where $\epsilon_{2}=-1,0$ or 1 according as the prime ideals of $k$ above 2 are inert, ramified or split in the quadratic extension $K / k)$. In particular, $\Pi_{K / k}(\{2\})=1$ if 2 is ramified in $K / k$. Finally, since $\kappa_{n, f, g}>0$, the right-hand side of (35) increases with $\rho_{K} \geq e^{2}$. Hence, for a given $n$ and and a given $\rho_{K} \geq 55>e^{4}$ we can easily compute the minima of the right-hand sides of (35) over all the pairs $(f, g)$ with $f \geq 1$ and $n \geq 1$ such that $f g$ divides $n$, and these minima increase with $\rho_{K} \geq 55>e^{4}$. This makes it easy to compute $\rho_{2 n}$ for any given entry $2 n$ in Table 6 .

In the same way, by using Point 2 of Lemma 8 we also obtain:

Theorem 23. Let $K$ be a not necessarily normal $C M$-field of degree $2 n \geq 2$ such that $\left(\zeta_{K} / \zeta_{k}\right)(s) \geq 0$ for $0<s<1$ and such that $k$ is abelian 7 Then (34) and (35) hold with $c=2$. In particular, for each entry $2 n$ in Table 6 , we have $h_{K}^{-}>1$ as soon as $\rho_{K} \geq \rho_{2 n}$.

\section{An APPLICATION OF THESE BETTER LOWER BOUNDS}

The aim of this section is to give an example showing the paramount usefulness of Theorem 22 when dealing with class group problems for various types of CMfields for which Theorem 9 is of less or no practical usefulness. In Lou5] we proved that if $K$ is a non-normal quartic CM-field, then

$$
h_{K}^{-} \geq \frac{\sqrt{d_{K} / d_{k}}}{12\left(\log \left(d_{K} / d_{k}\right)+0.052\right)^{2}}
$$

(notice that according to its proof, there is a misprint in the statement of the lower bound [Lou5, Corollary 15]). We will now improve upon this lower bound.

\footnotetext{
${ }^{7}$ For example, $K$ is a quaternion or a dihedral octic CM-field as in Lou3, and Lou5, Section 2.3.1].
} 
Lemma 24. Let $N$ be the normal closure of a non-normal quartic CM-field $K$. Hence, $N$ is a dihedral octic CM-field. Then,

$$
h_{N}^{-} \geq \frac{Q_{N} \Pi_{N / N^{+}}(\{2\}) \sqrt{d_{N} / d_{N^{+}}}}{4 e \pi^{4} B_{N^{+}}(\{2\}) \log d_{N}}
$$

(for $\left.\rho_{N} \geq 222\right)$, and

$$
B_{N^{+}}(\{2\}) \log d_{N} \leq\left(\log \left(d_{K} / d_{k}\right)+3\right)^{4} / 128 .
$$

Proof. To get (40), use (34) with $c=2$ (see Theorem 23). Let us now prove (41). Let $L_{1}=k, L_{2}$ and $L_{+}$be the three real quadratic subfields of $N^{+}$, the extension $N / L_{+}$being cyclic quartic, and let $\lambda_{1}, \lambda_{2}$ and $\lambda_{+}$be the constants $\kappa_{\chi}$ defined in (26) associated with the three quadratic characters $\chi$ of these three real quadratic fields. It is known that $L_{2}=\mathbf{Q}\left(\sqrt{d_{K} / d_{k}^{2}}\right)$ and that $d_{L_{2}}$ divides $d_{K} / d_{k}^{2}$. Since $d_{L_{+}}$divides the product $d_{L_{1}} d_{L_{2}}$ (for $N^{+} / \mathbf{Q}$ is biquadratic bicyclic), we conclude that $d_{L_{+}}$divides $d_{K} / d_{k}$. Upon using the bound $d_{N} \leq\left(d_{N} / d_{N^{+}}\right)^{2}=\left(d_{K} / d_{k}\right)^{4}$, we obtain (see 31):

$$
\begin{aligned}
128 B_{N^{+}} & (\{2\}) \log d_{N} \\
\leq & 4\left(\log d_{k}+\lambda_{1}\right)\left(\log \left(d_{K} / d_{k}^{2}\right)+\lambda_{2}\right)\left(\log \left(d_{K} / d_{k}\right)+\lambda_{+}\right) \log \left(d_{K} / d_{k}\right) \\
\leq & \left(\log \left(d_{K} / d_{k}\right)+\lambda_{1}+\lambda_{2}\right)^{2}\left(\log \left(d_{K} / d_{k}\right)+\lambda_{+}\right) \log \left(d_{K} / d_{k}\right) \\
& \left(\text { for } 4 a b \leq(a+b)^{2} \text { for } a \geq 0 \text { and } b \geq 0\right) \\
\leq & \left(\log \left(d_{K} / d_{k}\right)+\left(2 \lambda_{1}+2 \lambda_{2}+\lambda_{+}\right) / 4\right)^{4} \\
& \left(\text { for } a^{2} b c \leq((2 a+b+c) / 4)^{4} \text { for } a \geq 0, b \geq 0 \text { and } c \geq 0\right) .
\end{aligned}
$$

Finally, since either 2 splits in one of the three quadratic subfields of $k$, or 2 ramifies in at least two of the three quadratic subfields of $k$, we have $\left(2 \lambda_{1}+2 \lambda_{2}+\lambda_{+}\right) / 4 \leq$ $\left(4 \kappa_{3}+\kappa_{1}\right) / 4=2.830327 \cdots$.

Theorem 25. Let $K$ be a non-normal quartic CM-field. Assume that $\rho_{K} \geq 222$. Then,

$$
h_{K}^{-} \geq \frac{8 \Pi_{K / k}(\{2\}) \sqrt{d_{K} / d_{k}}}{\sqrt{e} \pi^{2}\left(\log \left(d_{K} / d_{k}\right)+3\right)^{2}} \geq \frac{\sqrt{d_{K} / d_{k}}}{C_{K}\left(\log \left(d_{K} / d_{k}\right)+3\right)^{2}}
$$

where

$$
C_{K}= \begin{cases}9 \sqrt{e} \pi^{2} / 32=4.57656 \cdots & \text { if } 2 \text { is not ramified in } K, \\ 3 \sqrt{e} \pi^{2} / 16=3.05104 \cdots & \text { if } 2 \text { is ramified in } K, \\ \sqrt{e} \pi^{2} / 8=2.03402 \cdots & \text { if } 2 \text { is totally ramified in } K .\end{cases}
$$

Proof. Let $N$ denote the normal closure of $K$. Then $N$ is a dihedral octic CM-field. Since $\zeta_{N} / \zeta_{N^{+}}=\left(\zeta_{K} / \zeta_{k}\right)^{2}$, it follows that $d_{N} / d_{N^{+}}=\left(d_{K} / d_{k}\right)^{2}, \Pi_{N / N^{+}}(\{2\})=$ $\left(\Pi_{K / k}(\{2\})\right)^{2}$ and $h_{N}^{-}=Q_{N}\left(h_{K}^{-}\right)^{2} / 2$. Using (40) and Lemma 24, we obtain the first lower bound for $h_{K}^{-}$.

As for the second lower bound, we use

$$
\Pi_{K / k}(\{2\})=\prod_{\mathcal{P}_{k} \mid(2)}\left(1-\frac{\chi\left(\mathcal{P}_{k}\right)}{N_{k / \mathbf{Q}}\left(\mathcal{P}_{k}\right)}\right)^{-1} \geq \begin{cases}1 & \text { if } 2 \text { is totally ramified in } K, \\ 2 / 3 & \text { if } 2 \text { is ramified in } K, \\ (2 / 3)^{2} & \text { in all cases, }\end{cases}
$$

where $\mathcal{P}_{k}$ ranges over the prime ideals of $k$ above the rational prime 2 and $\chi$ denotes the quadratic character associated with the extension $K / k$. 
Theorem 26. If the ideal class group of a non-normal quartic CM-field $K$ is of exponent $\leq 2$, then $h_{K}^{-} \leq 2^{15}$ and $d_{K} / d_{k} \leq 3 \cdot 10^{16}$.

Proof. We assume that $d_{K} / d_{k} \geq 3 \cdot 10^{9}$, which implies $\rho_{K}=d_{K}^{1 / 4} \geq\left(d_{K} / d_{k}\right)^{1 / 4} \geq$ 222 . Let $t$ denote the number of rational primes ramified in $k / \mathbf{Q}$ and let $T$ be the number of prime ideals ramified in $K / k$. Let $p_{1}=3 \leq p_{2}=3<p_{3}=5 \leq p_{4}=$ $5<p_{5}=7 \cdots$ be the nondecreasing sequence of all the odd primes, each one being repeated twice and set $\delta_{r}=\prod_{k=1}^{r} p_{k}$. In the same way, set $\tilde{p}_{1}=3 \leq \tilde{p}_{2}=3<\tilde{p}_{3}=$ $4 \leq \tilde{p}_{4}=4<\tilde{p}_{5}=5 \cdots$ (where for $k \geq 5$ we set $\tilde{p}_{k}=p_{k-2}$ ) and set $\tilde{\delta}_{r}=\prod_{k=1}^{r} \tilde{p}_{k}$. If 2 is not ramified in $K$, then $d_{K} / d_{k} \geq \delta_{t+T}$, whereas if 2 is ramified in $K$, then $d_{K} / d_{k} \geq \tilde{\delta}_{t+T}$. Now, assume that the ideal class group of a non-normal quartic CM-field $K$ is of exponent $\leq 2$. Then $h_{K}^{-} \leq 2^{t+T-2}$ (see [LYK, Corollary 17]). Now there are two cases to consider.

First, assume that 2 is not ramified in $K$. Using the lower bound (42) (which is an increasing function of $d_{K} / d_{k}$ ), we obtain

$$
2^{t+T-2} \geq h_{K}^{-} \geq \frac{\sqrt{\delta_{t+T}}}{C_{K}\left(\log \left(\delta_{t+T}\right)+3\right)^{2}} \text { with } C_{K}=9 \sqrt{e} \pi^{2} / 32,
$$

which implies $t+T \leq 16, h_{K}^{-} \leq 2^{14}$ and $d_{K} / d_{k} \leq 1.5 \cdot 10^{16}$, by using (42).

Second, assume that 2 is ramified in $K$. Using the lower bound (42) (which is an increasing function of $d_{K} / d_{k}$ ), we obtain

$$
2^{t+T-2} \geq h_{K}^{-} \geq \frac{\sqrt{\tilde{\delta}_{t+T}}}{C_{K}\left(\log \left(\tilde{\delta}_{t+T}\right)+3\right)^{2}} \quad \text { with } C_{K}=3 \sqrt{e} \pi^{2} / 16,
$$

which implies $t+T \leq 17, h_{K}^{-} \leq 2^{15}$ and $d_{K} / d_{k} \leq 2.8 \cdot 10^{16}$, by using (42).

Remarks 27.

(1) If we use (13) with $c=2$ (see Theorem 11) and Lemma 24] we obtain the following lower bounds for relative class numbers of non-normal quartic CM-fields:

$$
h_{K}^{-} \geq \frac{\sqrt{d_{K} / d_{K^{+}}}}{C_{K}\left(\log \left(d_{K} / d_{K^{+}}\right)+5 \kappa_{1} / 4\right)^{2}} \quad \text { where } C_{K}=\sqrt{e} \pi^{2} / 2=8.13611 \cdots .
$$

Using this lower bound, we would only obtain that if the ideal class group of a non-normal quartic CM-field $K$ is of exponent $\leq 2$, then $h_{K}^{-} \leq 2^{16}$ and $d_{K} / d_{K^{+}} \leq$ $9 \cdot 10^{17}$, a 30 -fold less satisfactory bound than the previous one.

(2) If we had used (39), we would only have obtained that if the ideal class group of a non-normal quartic CM-field $K$ is of exponent $\leq 2$, then $h_{K}^{-} \leq 2^{17}$ and $d_{K} / d_{k} \leq 10^{19}$, a 333 -fold less satisfactory bound than the previous one (and in fact a bound of no practical use).

(3) The desire to determine all the non-normal quartic CM-fields and all the dihedral octic CM-fields with ideal class groups of exponents $\leq 2$ has been a continuous incentive to obtain here as good as possible lower bounds for relative class numbers of CM-fields. These determinations have now been completed and can be found in $[\mathrm{LYK}$. 


\section{REFERENCES}

[Arn] S. Arno, The imaginary quadratic fields of class number 4, Acta Arith. 60 (1992), 321-324. MR 93b:11144

[Bak1] A. Baker, A remark on the class number of quadratic fields, Bull. London Math. Soc. 1 (1966), 98-102. MR 39:2723

[Bak2] A. Baker, Imaginary quadratic fields of class number 2, Ann. of Math. 94 (1971), 139-152. MR 45:8631

[Bes] S. Bessassi, Bounds for the degrees of CM-fields of class number one, Acta Arith. 106 (2003), 213-245.

[BP] E. Brown and C. J. Parry, The imaginary bicyclic biquadratic fields with class number 1, J. Reine Angew. Math. 266 (1974), 118-120. MR 49:4974

[FM] R. Foote and V. K. Murty, Zeros and poles of Artin L-series, Math. Proc. Cambridge Philos. Soc. 105 (1989), 5-11. MR 89k:11109

[HaHu] K. Hardy and R. H. Hudson, Determination of all imaginary cyclic quartic fields with class number 2, Trans. Amer. Math. Soc. 311 (1989), 1-55. MR 89f:11148

[HH] K. Horie and M. Horie, CM-fields and exponents of their ideal class groups, Acta Arith. 55 (1990), 157-170. MR 91k:11098

[HJ] J. Hoffstein and N. Jachnowitz, On Artin's conjecture and the class number of certain CM-fields, I and II, Duke Math. J 59 (1989), 553-563 and 565-584. MR 90h:11104

[Hof] J. Hoffstein, Some analytic bounds for zeta functions and class numbers, Invent. Math. $\mathbf{5 5}$ (1979), 37-47. MR 80k:12019

[Lan] S. Lang, Algebraic Number Theory, Springer-Verlag, Graduate Texts in Math. 110, Second Edition, 1994. MR 95f:11085

[LLO] F. Lemmermeyer, S. Louboutin and R. Okazaki, The class number one problem for some non-abelian normal CM-fields of degree 24, J. Théorie des Nombres de Bordeaux 11 (1999), 387-406. MR 2001j:11104

[LO] S. Louboutin and R. Okazaki, The class number one problem for some non-abelian normal CM-fields of 2-power degrees, Proc. London Math. Soc. (3) 76 (1998), 523-548. MR 99c:11138

[Lou1] S. Louboutin, Majorations explicites de $|L(1, \chi)|$, C. R. Acad. Sci. Paris 316 (1993), 11-14. MR 93m:11084

[Lou2] S. Louboutin, Lower bounds for relative class numbers of CM-fields, Proc. Amer. Math. Soc. 120 (1994), 425-434. MR 94d:11089

[Lou3] S. Louboutin, Determination of all quaternion octic CM-fields with class number $2, \mathrm{~J}$. London Math. Soc. 54 (1996), 227-238. MR 97g:11122

[Lou4] S. Louboutin, A finiteness theorem for imaginary abelian number fields, Manuscripta Math. 91 (1996), 343-352. MR 97f:11089

[Lou5] S. Louboutin, The class number one problem for the non-abelian normal CM-fields of degree 16, Acta Arith. 82 (1997), 173-196. MR 98j:11097

[Lou6] S. Louboutin, Majorations explicites du résidu au point 1 des fonctions zêta des corps de nombres, J. Math. Soc. Japan 50 (1998), 57-69. MR 99a:11131

[Lou7] S. Louboutin, Explicit bounds for residues of Dedekind zeta functions, values of $L$-functions at $s=1$, and relative class numbers, J. Number Theory 85 (2000), 263-282. MR 2002i: 11111

[Lou8] S. Louboutin, Explicit upper bounds for residues of Dedekind zeta functions and values of $L$-functions at $s=1$, and explicit lower bounds for relative class numbers of CM-fields, Canad J. Math. 53 (2001), 1194-1222. MR 2003d:11167

[Lou9] S. Louboutin, Majorations explicites de $|L(1, \chi)|$ (quatrième partie), C. R. Acad. Sci. Paris 334 (2002), 625-628.

[LPP] H. W. Lenstra, J. Pila and C. Pomerance, A hyperelliptic smoothness test, II, Proc. London Math. Soc. 84 (2002), 105-146.

[LYK] S. Louboutin, Y.-S. Yang and S.-H. Kwon, The non-normal quartic CM-fields and the dihedral octic CM-fields with ideal class groups of exponent $\leq 2$, Preprint (2000).

[Mur1] M. Ram Murty, An analogue of Artin's conjecture for abelian extensions, J. Number Theory 18 (1984), 241-248. MR 85j:11161

[Mur2] V. K. Murty, Class numbers of CM-fields with solvable normal closure, Compositio Math. 127 (2001), 273-287. MR 2003a:11147 
[MW] H. L. Montgomery and P. J. Weinberger, Note on small class numbers, Acta Arith. 24 (1974), 529-542. MR 50:9841

[Odl] A. Odlyzko, Some analytic estimates of class numbers and discriminants, Invent. Math. 29 (1975), 275-286. MR 51:12788

[Oes] J. Oesterlé, Nombres de classes des corps quadratiques imaginaires, Séminaire Bourbaki, 36e année, 1983-84, exposé 631. Astérisque 121-122 (1985), 309-323. MR 86k:11064

[Oka] R. Okazaki, Inclusion of CM-fields and divisibility of relative class numbers, Acta Arith. 92 (2000), 319-338. MR 2001h:11138

[Pin1] J. Pintz, On Siegel's theorem, Acta Arith. 24 (1974), 543-551. MR 49:2595

[Pin2] J. Pintz, Elementary methods in the theory of $L$-functions, VIII, Real zeros of real $L$ functions, Acta Arith. 33 (1977), 89-98. MR 58:5551g

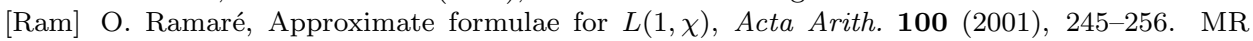
2002k:11144

[Sie] C. L. Siegel, Über die Classenzahl quadratischer Zahlkörper, Acta Arith. 1 (1935), 83-86.

[Sta1] H. M. Stark, A complete determination of the complex quadratic fields of class number 1 , Michigan Math. J. 14 (1967), 1-27. MR 36:5102

[Sta2] H. M. Stark, On complex quadratic fields with class number two, Math. Comp. 29 (1975), 289-302. MR 51:5548

[Sta3] H. M. Stark, Some effective cases of the Brauer-Siegel Theorem, Invent. Math. 23 (1974), 135-152. MR 49:7218

[Uch] K. Uchida, Imaginary abelian number fields with class number one, Tôhoku Math. J. 24 (1972), 487-499. MR 48:269

[Was] L. C. Washington, Introduction to Cyclotomic Fields, Springer-Verlag, Graduate Texts in Math. 83, Second Edition, 1997. MR 97h:11130

[YK] H.-S. Yang and S.-H. Kwon, The non-normal quartic CM-fields and the octic dihedral CMfields with relative class number two, J. Number Th. 79 (1999), 175-193. MR 2000h:11117

Institut de Mathématiques de Luminy, UPR 9016, 163 avenue de Luminy, Case 907, 13288 Marseille Cedex 9, France

E-mail address: loubouti@iml.univ-mrs.fr 